\title{
POTENCIAL DE REUTILIZACIÓN DEL EFLUENTE DE LA PLANTA DE TRATAMIENTO DE AGUAS RESIDUALES DE NÁTAGA EN CULTIVO DE CACAO (Theobroma cacao L.)
}

\section{POTENTIAL REUSE OF EFFLUENT FROM THE NÁTAGA MUNICIPALITY WASTEWATER TREATMENT PLANT FOR THE CROP OF COCOA (Theobroma cocoa L.)}

\author{
Eduardo Valencia1, Renso Alfredo Aragón², Jonathan Romero³
}

\begin{abstract}
1 M.Sc. Profesor Asociado, Universidad Surcolombiana, Departamento de Ingeniería Agrícola, Neiva, Huila, Colombia, eduvale@ usco.edu.co. Autor para correspondencia. ${ }^{2}$ Ing. Agrícola; Grupo de Investigación Agroindustria USCO, Universidad Surcolombiana; Neiva-Huila-Colombia; renso733@hotmail.com ${ }^{3}$ Ing. Agrícola; Centro de Investigación en Ciencias y Recursos GeoAgroAmbientales - CENIGAA; Neiva - Huila - Colombia; jonathan.romero@cenigaa.org
\end{abstract}

Rev. Act. E Div..Cient. Año 15(1): 77 - 86, 2012

\section{RESUMEN}

El trabajo consistió en determinar el potencial de reutilización del efluente de la Planta de Tratamiento de Aguas Residuales (PTAR) del municipio Nátaga, departamento Huila, Colombia, con fines de irrigación para un cultivo de cacao (Theobroma cacao L.). Este efluente es vertido a la Quebrada El Pueblo, creando problemas de contaminación y de riesgos de salud pública. Aplicando vigilancia tecnológica, se elaboró el estado del arte y recolección de información, para caracterizar el efluente y evaluar la PTAR; se calculó el balance hídrico del cultivo y los beneficios potenciales de la reutilización en riego de estas aguas residuales. El efluente de la PTAR pertenece a la clase $\mathrm{C} 2 \mathrm{~S} 1(\mathrm{CE}=0,54 \mathrm{dS} / \mathrm{m}$, RAS $=1,5)$, con peligro de salinización medio y de alcalinización bajo para agua de riego; por su riesgo microbiológico es categoría $\mathrm{B}(\mathrm{CF}=$ $105 \mathrm{NMP} / 100 \mathrm{~mL}, \mathrm{HH}=21 \mathrm{HH} / \mathrm{L})$, exigiendo adecuado manejo del riego, que minimice los riesgos de salud pública. La zona de influencia tiene déficit hídrico durante todo el año y el efluente constituye un recurso potencial para el cultivo de cacao, aportando en promedio 223.720 mªño de agua, macronutrientes $N(7.273 \mathrm{~kg} / \mathrm{año}), P(839 \mathrm{~kg} / \mathrm{año})$, $\mathrm{K}(2.797 \mathrm{~kg} / \mathrm{año})$ y lodos (958 kg/año de materia seca); con el efluente en verano, se puede regar en promedio 19ha de cacao y, en invierno, 27ha. Se recomienda el riego del cacao con el efluente de la PTAR, pero se debe tener cuidado con los efectos sobre el suelo, la planta y la salud pública.

Palabras clave: Aguas residuales, reutilización de agua, riego, cacao.
SUMMARY

The project consisted in the determination of the potential reuse of the effluent from the Nátaga municipality Wastewater Treatment Plant (WWTP) for irrigation purpose in cocoa cultivations (Theobroma cacao L.). The effluent is discharged to the "El Pueblo" river, generating pollution problems and public health risks. By means of technological surveillance, the state of art was elaborated and the information, in order to characterize and evaluate the WWTP effluent, collected. The crops water balance and the potential benefits of reuse in irrigation of wastewater was calculated. The effluent from the WWTP belongs to the C2S1 class $(E C=0.54 \mathrm{dS}$ $\mathrm{m}^{-1}, \mathrm{SAR}=1.5$ ) with a medium risk of salinization and a low risk of alkalinization for water use in irrigation. The microbiological risk assessment is considered in category $\mathrm{B}$ ( $\mathrm{CF}=105 \mathrm{MPN} / 100 \mathrm{~mL}, \mathrm{HH}=21 \mathrm{HH} / \mathrm{L}$ ), demanding an adequate irrigation management to minimize public health risks. The area of influence has a water deficit throughout the year and the effluent is a potential resource for the cultivation of cacao, providing an $223,720 \mathrm{~m}^{3}$ year-1 average of water, macronutrients $\mathrm{N}$ (7273 kg year-1), P (839 kg year-1), K $(2,797 \mathrm{~kg}$ year-1) and sludge (958 kg year-1 of dry matter). The average effluent for irrigation purpose in the dry season can be for 19 ha and 27 ha in the rainy season. It is recommended to irrigate cocoa cultures with the effluent from the WWTP, being careful with the effects on soil, plant and public health.

Key words: Wastewater, water reuse, irrigation, cocoa. 


\section{INTRODUCCIÓN}

El concepto de manejo integral del recurso hídrico implica la gestión eficaz basada en el ahorro, la reutilización y la no contaminación del agua, la implementación de tecnologías e infraestructura multipropósito, en una planificación sostenible del territorio y una gestión integrada de las cuencas hidrográficas (Morató et al. 2006; O'Connor et al. 2010).

Por consiguiente, la OMS (Organización Mundial de la Salud) (2006) y la FAO (Food and Agriculture Organization of the United Nations) (2011), están fomentando el uso de las aguas residuales (AR) de origen doméstico en la agricultura, pero reconocen los riesgos que generan para la salud y el ambiente (Pescod, 1992; Kiziloglu et al. 2008; Segal et al. 2011; Cirelli et al. 2012).

Por lo tanto, la reutilización de aguas residuales tratadas (ART) para riego agrícola, se convierte en una solución técnica para minimizar la degradación del suelo y restaurar el contenido de nutrientes del mismo (Kiziloglu et al. 2008). Adicionalmente, Qadir et al. (2007) plantean el uso de recursos hídricos no convencionales (aguas subterráneas con condiciones salinas, aguas de drenaje agrícola y ART, entre otros), para enfrentar el problema de la seguridad alimentaria de las grandes ciudades, teniendo precaución con los problemas de salinidad y de sodicidad asociados al suelo. Pero, Segal et al. (2011) indican que la reutilización de ART para riego de cultivos agrícolas incrementa la susceptibilidad de los cultivos al estrés osmótico y aumenta el potencial de contaminación de aguas subterráneas.

Cuando los cultivos son regados con ART, los primeros y los principales cambios, se encuentran en los parámetros de salinidad y de sodicidad del suelo (Marques et al. 2009). Por otro lado, en proyectos de reutilización de AR para riego, las directrices de la OMS son las indicadas para la calidad microbiológica del agua. El objetivo principal del tratamiento de las AR debe ser la eliminación de los organismos patógenos (León, 1995; OMS, 2006), como los relacionados con excretas (bacteria Escherichia coli, Vibro chorelae, Salmonella spp., Shigella spp.), helmintos (Ascaris, Ancylostoma, Necator, Hymenolepis, Strongyloides, Toxocara, Trichuris, Taenia spp.), trematodos, protozoarios, virus, los relacionados con patógenos transmitidos por vectores, irritaciones en la piel y químicos. En lo que respecta a los criterios de calidad físicoquímica de agua para riego, las directrices de la FAO (1999) proponen la salinidad y la alcalinización de los suelos como parámetros.

De acuerdo con Silva et al. (2008), las nuevas guías de uso de AR, de excretas y de aguas grises, publicadas en 2006, sirven como herramienta en el manejo preventivo de AR en agricultura e, incluyen, análisis microbiano con recolección de información relacionada con patógenos, no da valores para patógenos virales, bacteriales o protozoarios, sólo para $\mathrm{HH}(\leq 1 / \mathrm{L})$ en riego con o sin restricción; para el riego por goteo en cultivos de alto crecimiento, no da recomendación alguna y comprende medidas de control para la protección de la salud. Estas nuevas directrices reafirman las recomendaciones dadas por la OMS en 1989, con criterios de calidad microbiológica, más accesibles de cumplir por los países pobres y en vía de desarrollo (Veliz et al. 2009).

En ese orden de ideas, la reutilización del ART puede exigir un cambio en las especies vegetales utilizadas para el cultivo, la modificación de las dosis de fertilizantes, la remodelación del sistema de riego, la adopción de precauciones para proteger a los trabajadores agrícolas y la salud de los consumidores (Mujeriego, 1990).

Se han desarrollado varias investigaciones en el orden mundial para conocer los potenciales beneficios y los problemas de los proyectos de reutilización de ART, para riego de cultivos agrícolas y para trabajos de investigación aplicada, interesados en aumentar los recursos hídricos de zonas áridas (Nunes et al. 2005; Fasciolo et al. 2005; Rutkowski et al. 2006; Hassanli et al. 2008).

Medeiros et al. (2005) encontraron que ART, con filtros de arena, mejoran las características del suelo, como el pH, el porcentaje de materia orgánica (M.O.), el potasio (K), el calcio (Ca) y el magnesio $(\mathrm{Mg})$, pero incrementa los problemas de salinidad. Umaña (2007) evaluó rendimientos del cultivo de maíz regado con ART y con aguas de riego; no encontró diferencia significativa en rendimientos, pero indica que el riego con AR puede ahorrar el uso de fertilizantes. Kiziloglu et al. (2008) evaluaron las mejoras en la fertilidad del suelo sin afectar la calidad del mismo y de las plantas al aplicar riego con ART. Los resultados indican que el riego con ART afecta, significativamente, las propiedades químicas del suelo en los primeros $30 \mathrm{~cm}$ de profundidad y el contenido de nutrientes en las plantas después de la cosecha. El experimento no arrojó afectaciones por metales pesados ni excesos de sales. Se concluye, que las AR domésticas sin tratar, se pueden usar con confianza para riego agrícola a corto plazo y con tratamiento primario, en agricultura sustentable, a largo plazo. De igual forma, en Argentina, se comparó el rendimiento de tubérculos (topinamburregados) con AR y con agua subterránea, obteniendo mejor rendimiento con el primer tipo de riego (Lelio et al. 2009).

De otra parte, Marques et al. (2009) evaluaron los efectos de la salinidad y la sodicidad del suelo en una plantación de caña de azúcar en condiciones tropicales, regada 16 meses con ART, en Sao Pablo, Brasil. Los resultados indican 
diferencias significativas en el incremento de porcentaje de sodio intercambiable, dispersión de arcillas, tasa de adsorción de sodio y conductividad eléctrica; sin embargo se concluye, que el incremento del contenido de sodio en el suelo está más asociado a la frecuencia de riego con ART que a la cantidad de ART aplicada al cultivo. Por lo tanto, se recomienda estudiar los procesos de remediación del suelo para poder implementar la reutilización de ART, como solución técnica.

Actualmente, el municipio de Nátaga, se proyecta como productor potencial de cacao, en el departamento del Huila. Particularmente, se ha presentado un proyecto para la construcción del distrito de riego a pequeña escala de Nátaga "Asodriana", para abastecer de agua 120 ha de cacao.

Por consiguiente, este estudio presenta el potencial de reutilización del efluente de la PTAR del municipio de Nátaga: Agua y nutrientes para el cultivo de cacao, como una alternativa ambiental y productiva, considerada como un tratamiento terciario para la PTAR, donde el efluente pasa de ser un contaminante a convertirse en un recurso.

\section{MATERIALES Y MÉTODOS}

Localización: El municipio de Nátaga está localizado en la región Sur - Occidental del Departamento del Huila, su cabecera municipal se encuentra a $2^{\circ}$ y $34^{\prime}$ de latitud Norte y $75^{\circ} 49^{\prime}$ de longitud Oeste, a una distancia de $120 \mathrm{~km}$ de la capital, en las laderas de una ramificación de la cordillera central. Tiene una superficie de $132,7 \mathrm{~km}^{2}$, perteneciendo 44,6 ha, al perímetro urbano. Se encuentra a una altitud de $1.480 \mathrm{msnm}$, donde el 3,55\% del territorio corresponde a piso térmico cálido, con una temperatura promedio de $24^{\circ} \mathrm{C}$; el 53,88\% corresponde a Clima Medio Húmedo $(\mathrm{MH})$, con temperaturas entre $18^{\circ}$ y $24^{\circ} \mathrm{C}$; el $42,56 \%$ pertenece a Clima Medio muy Húmedo (MMH), con alturas superiores a los 2.000 msnm. La PTAR, se encuentra ubicada a unos 300 $\mathrm{m}$ de la Alcaldía municipal y el distrito de riego, se situaría contiguo a la PTAR.

Caracterización de los recursos geofísicos de la zona de estudio: Se realizó la consulta de casos de estudio y del estado del arte, lo cual, consistió en revisión de artículos científicos y de bases de datos especializadas, utilizando herramientas de vigilancia tecnológica. Se hizo el reconocimiento y el análisis del área de estudio; recolección de información del diseño y monitoreo de la PTAR; se tomaron datos e información sobre las unidades del sistema de tratamiento, población, caudales y disposición del efluente; se analizaron los resultados fisicoquímicos y microbiológicos de una caracterización del afluente y efluente y, finalmente, se recolectó información climatológica de la zona de estudio, como precipitación, evaporación, temperatura, las propiedades físicas y químicas del suelo.

Descripción de la PTAR y cálculo de eficiencias: Para la caracterización del efluente, se realizaron muestreos puntuales del afluente y del efluente de la PTAR, el 23 de marzo de 2010, en horas de la mañana y analizados en el Laboratorio de Aguas de la Universidad Surcolombiana: Fisicoquímico, demanda biológica de oxígeno $\mathrm{DBO}_{5}$ (método de Winkler), sólidos suspendidos SS (método gravimétrico), grasas y aceites G y A (método partición gravimétrica) y microbiológico, coliformes totales CT y coliformes fecales CF (método de tubos múltiples) y también algunos datos fueron tomados de la literatura. Se determinó la eficiencia de la PTAR, que permitiera la caracterización del efluente y su categorización como agua para riego, de acuerdo con las directrices de la FAO (1999) y de la OMS (2006) y la descripción de las unidades de tratamiento. Para el cálculo de la eficiencia se empleó la fórmula (1):

$$
\% \operatorname{Re} \text { moción }=\frac{\text { Concentración Af }- \text { Concentración Ef }}{\text { Concentración Af }} * 100
$$

Af : Afluente; $E f$ : Efluente

Determinación del balance hídrico: Para la caracterización del clima, se seleccionó la estación climatológica Nátaga, considerando criterios de representatividad y de homogeneidad sobre el área de estudio y por encontrarse dentro del área de influencia del proyecto; debido a que sólo cuenta con registros pluviométricos, también se utilizó la estación Escuela Agrícola La Plata, con registros completos de los elementos del clima. La información climatológica para el estudio corresponde a datos multianuales, del periodo 1998 - 2008. Se determinó el balance hídrico del cultivo para la zona de estudio, mediante la precipitación efectiva por el método USDA Soil Conservation Service (Jaramillo, 2008), la evapotranspiración del cultivo, por el método de tanque evaporímetro (Allen et al. 1998), la demanda de riego neta y la necesidad de riego.

Potencialidad de reutilización del efluente de la PTAR: La determinación del volumen de agua disponible para reutilizar en riego del cultivo de cacao, se realizó con base en la población de Nátaga, empleando el método geométrico para el cálculo de la población, con tasa de crecimiento de 2,8 y un periodo de 20 años. Se determinó el área a regar, por medio de la metodología de Jaramillo (2008) y los aportes en nutrientes: Nitrógeno $(\mathrm{N})$, fósforo $(\mathrm{P})$ y potasio $(\mathrm{K})$ para el cultivo de cacao, mediante el cálculo de los recursos aportados por el efluente; se utilizaron las expresiones (2) y (3): 
Cantidad Nutrientes $=$ Población $* C a_{p c}$

$\mathrm{Ca}_{\mathrm{pc}}=$ carga per cápita de Nutriente.

$$
C a_{p c}=\operatorname{Dot}^{*} C
$$

Dot $=$ Dotación $; \mathrm{C}=$ Concentración de nutriente.

\section{RESULTADOS Y DISCUSIÓN}

Descripción de la Planta de Tratamiento: El municipio de Nátaga, en el 2006, contaba con 6.785 habitantes, de los cuales, 4.550 pertenecían a la zona rural y 2.235, a la zona urbana; es de vocación agrícola y su principal cultivo es el café. Cuenta con los servicios públicos básicos, la dotación estimada para el 2010 es 330L/hab-d y se contempla una disminución gradual hasta $150 \mathrm{~L} / \mathrm{hab}-\mathrm{d}$, para el 2026. Su PTAR tiene una cobertura del $100 \%$ y recibe un caudal de $4,66 \mathrm{~L} / \mathrm{s}$.

La figura 1 muestra el esquema general, que incluye la propuesta de reutilización del efluente en un cultivo de cacao, la cual, está conformada por las siguientes unidades: Tratamiento preliminar, que consta de un canal de aproximación, un vertedero de excesos, dos rejillas y dos desarenadores en paralelo y una canaleta Parshall; tratamiento secundario, conformado por cuatro UASB (Upflow anaerobic sludge blanket) y dos Filtros Anaerobios de Flujo Ascendente (FAFA), con lechos filtrantes plásticos; los lodos son llevados a cuatro lechos de secado; actualmente, el efluente es vertido a la Quebrada El Pueblo. Es pertinente aclarar que el vertedero de excesos se utiliza, principalmente, en épocas de lluvia, para aguas de escorrentía superficial y se vierten en la quebrada. En este sentido, se propone construir un pequeño reservorio que almacene el agua de escorrentía y que luego sea bombeada al canal de aproximación, para su respectivo tratamiento; de esta manera, se evita la posible fuente de contaminación que se presenta cuando hay demasiada escorrentía superficial.

\section{Evaluación del sistema}

Caracterización del Efluente y Eficiencia de la PTAR: Las tablas 1 y 2 presentan los resultados de la eficiencia de la PTAR y de las características del efluente, respectivamente.

El efluente de la PTAR de Nátaga, según la clasificación propuesta por Mara \& Cairncross (1990) y de acuerdo con sus valores de conductividad eléctrica $(\mathrm{CE})=0,54 \mathrm{dS} / \mathrm{m}$ yla relación de adsorción de sodio (RAS) = 1,5 se clasifica C2S1, agua de buena calidad para riego, con peligro de salinización

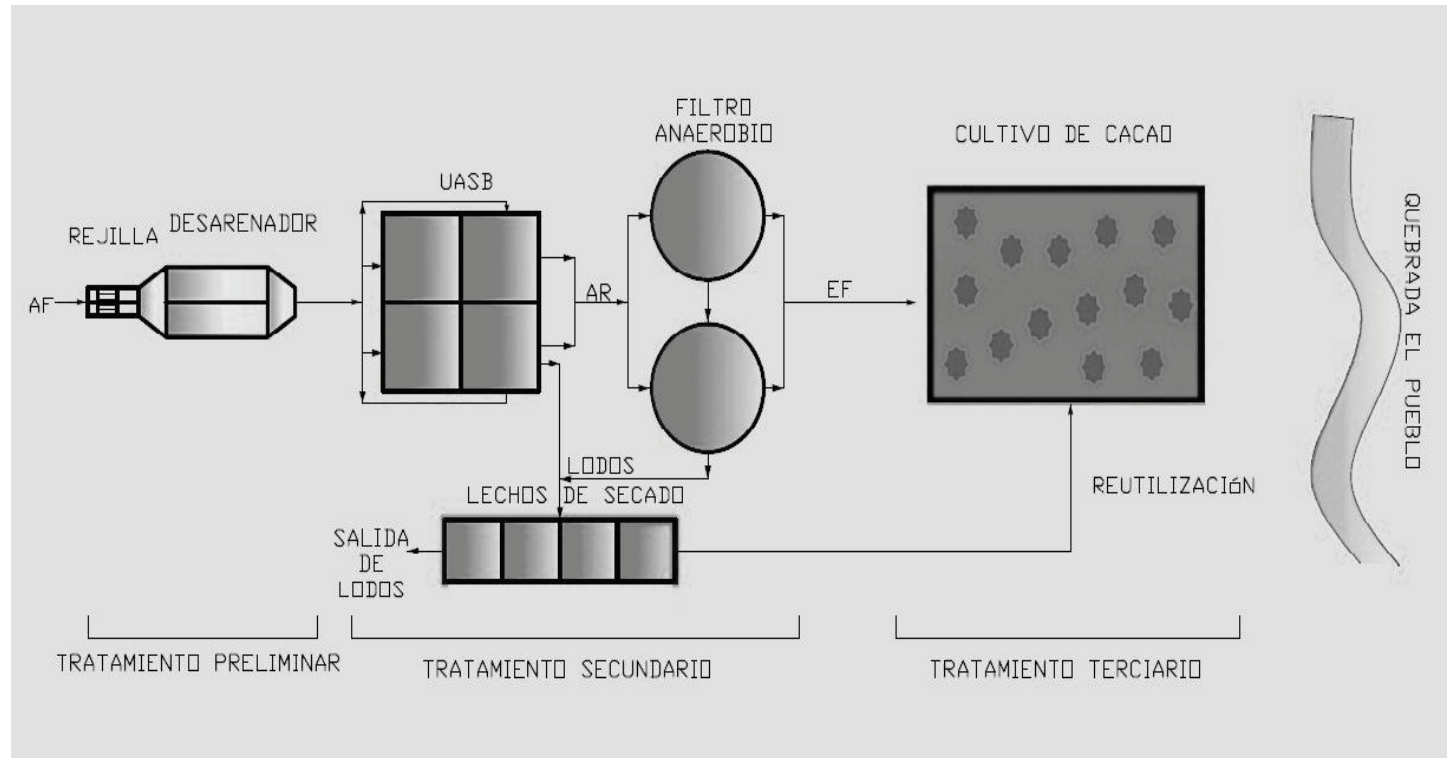

Figura 1. Esquema general de la PTAR de Nátaga incluyendo reutilización. 
Tabla 1. Eficiencia de la PTAR.

\begin{tabular}{|l|c|c|c|}
\hline PARÁMETRO & AFLUENTE & EFLUENTE & \% REMOCIÓN \\
\hline DBO $(\mathrm{mg} / \mathrm{L})$ & 170 & 39 & 77 \\
\hline SS $(\mathrm{mg} / \mathrm{L})$ & 153 & 27 & 82 \\
\hline G y A (mg/L) & 16 & 2,3 & 86 \\
\hline N (mg/L) & $30 \mathrm{~b}$ & 26 a & 13 \\
\hline P (mg/L)b & 6 & 3 & 50 \\
\hline CT NMP/100mL) & $10^{10}$ & $10^{6}$ & 99,99 \\
\hline CF (NMP/100mL) & $10^{9}$ & $10^{5}$ & 99,99 \\
\hline HH (No./L) & 21 & 21 & 0 \\
\hline
\end{tabular}

Fuente: aValencia, 2003; bRAS, 2000.

Nota: CT: Coliformes Totales; G y A: Grasas y Aceites.

Tabla 2. Caracterización del Efluente.

\begin{tabular}{|l|c|c|}
\hline PARÁMETRO & UNIDADES & VALOR \\
\hline $\mathrm{DBO}_{5}$ & $\mathrm{mg} / \mathrm{L}$ & 39 \\
\hline $\mathrm{SS}$ & $\mathrm{mg} / \mathrm{L}$ & 27 \\
\hline $\mathrm{G} \mathrm{y}$ & $\mathrm{mg} / \mathrm{L}$ & 2,3 \\
\hline $\mathrm{Na}^{\mathrm{a}}$ & $\mathrm{mg} / \mathrm{L}$ & 26 \\
\hline $\mathrm{Pb}$ & $\mathrm{mg} / \mathrm{L}$ & 3 \\
\hline $\mathrm{K}^{\mathrm{a}}$ & $\mathrm{mg} / \mathrm{L}$ & 10 \\
\hline $\mathrm{CT}$ & $\mathrm{NMP} / 100 \mathrm{~mL}$ & $10^{6}$ \\
\hline $\mathrm{CF}$ & $\mathrm{NMP} / 100 \mathrm{~mL}$ & $10^{5}$ \\
\hline $\mathrm{HH}$ & $\mathrm{No} / \mathrm{L}$ & 21 \\
\hline $\mathrm{CE} \mathrm{a}^{\mathrm{a}}$ & $\mathrm{dS} / \mathrm{m}$ & 0,54 \\
\hline $\mathrm{RAS}^{\mathrm{a}}$ & & 1,5 \\
\hline
\end{tabular}

Fuente: aValencia, 2003; bRAS, 2000.

medio y de alcalinización bajo; de menor calidad a las aguas de la mayoría de los tramos del río Cauca, que son C1S1 aguas excelentes para riego e igual a la de otros tramos del mismo y, a las aguas subterráneas, utilizadas en el Valle del Cauca, que son C2S1 y según las Directrices de la FAO (1999), presenta un Grado de Restricción Moderado, requiriéndose un cuidado gradualmente mayor en la selección del cultivo y de las alternativas de manejo del mismo y del suelo, como el drenaje agrícola, para alcanzar el potencial máximo de rendimiento. Este peligro de salinización, se puede convertir en un impacto ambiental negativo, debido a los problemas que puede presentar por procesos de infiltración y de acumulación de sales en el tiempo.

Respecto a la calidad microbiológica, por el valor de coliformes fecales $(\mathrm{CF})=10^{5} \mathrm{NMP} / 100 \mathrm{~mL}$, el efluente pertenece a la Categoría $B$, que puede ser empleado para riego de cultivos, como: cereales, industriales y forrajeros, praderas y árboles (OMS, 2006), por tanto, el efluente se puede utilizar para riego del cultivo de cacao; sin embargo, 
según la OMS existe riesgo microbiológico que puede afectar la salud pública, fundamentalmente, por el alto contenido de huevos de helmintos $(\mathrm{HH})=21 \mathrm{HH} / \mathrm{L}$; el grupo expuesto son los agricultores y se recomienda el riego por microaspersión para minimizarlo. La densidad de $\mathrm{HH}$, se tomó teniendo como antecedente el valor encontrado en el afluente, en un estudio realizado por Valencia (2003) y se asumió que no había remoción, teniendo en cuenta que, según el RAS (2000), las remociones esperadas para el tipo de sistema de tratamiento del municipio de Nátaga son muy bajas.

Para asegurar la calidad del efluente y poder utilizarlo en riego del cultivo de cacao, la PTAR de Nátaga debe funcionar de acuerdo con su diseño, lo que exige una correcta operación y mantenimiento. Las remociones de DBO del $77 \%$ y SS del $82 \%$ (Tabla 1), indican que la PTAR es eficiente en la remoción de contaminantes, eficiencias que se pueden aumentar mejorando su operación; no obstante, microbiológicamente, no se esperan remociones mayores de $\mathrm{CF}$ y HH por el tipo de unidades que conforman la PTAR. La evaluación microbiológica del AR constituye un factor importante a la hora de restringir la forma de regar, pues se pueden presentar enfermedades que se propagan, cuando se realiza mal manejo de AR, como las diarreas, la tifoidea y la paratifoidea, el cólera, la hepatitis infecciosa, la amibiásis, giardiasis, entre otras (Sáenz, 1994).
Componente Climático de la zona de estudio: En la zona donde está ubicada la PTAR, se proyecta el distrito de riego "Asodriana"; la temperatura media mensual multianual varía ligeramente durante el año, con un valor mínimo de $21,2^{\circ} \mathrm{C}$, en julio y máximo de $22,3^{\circ} \mathrm{C}$, en marzo. La distribución de la precipitación mensual multianual presenta dos picos extremos, con dos periodos de lluvias comprendidos entre marzo a junio y de octubre a diciembre y un periodo de transición con precipitaciones menores entre enero y febrero; enero es el más seco, con un promedio de $183 \mathrm{~mm}$ y mayo el de mayor valor mensual promedio, con $416 \mathrm{~mm}$. La mayor evaporación media se presenta en septiembre, con un valor de $111,9 \mathrm{~mm}$ y, la menor, en abril, con 81,7mm (Tabla 3).

Balance Hídrico: La tabla 3 y la figura 2 muestran que durante todo el año la zona donde se encuentra la PTAR presenta déficit hídrico, convirtiéndose el efluente en un recurso importante para suplir, en parte, las necesidades de riego. Esta situación posibilita la consecución de agua suplementaria, como en el caso citado por Mujeriego (1990), donde las mayores posibilidades de aumentar los recursos hidráulicos es mediante la utilización de AR regenerada, es decir, conseguir agua adicional para el estado de California.

Pero se debe tener en cuenta que la reutilización de esta agua puede provocar efectos ambientales adversos, entre

Tabla 3. Balance Hídrico Mensual Multianual.

\begin{tabular}{|l|c|c|c|c|}
\hline \multicolumn{1}{|c|}{ MESES } & $\begin{array}{c}\text { PRECIPITACIÓN } \\
\text { EFECTIVA } \\
\text { (mm/mes) }\end{array}$ & $\begin{array}{c}\text { EVAPORACIÓN } \\
\text { (mm/mes) }\end{array}$ & $\begin{array}{c}\text { USO CONSUNTIVO } \\
\text { (mm/mes) }\end{array}$ & $\begin{array}{c}\text { DEMANDA DE RIEGO } \\
\text { NETA (mm/mes) }\end{array}$ \\
\hline Enero & 21,13 & 95,70 & 100,49 & 79,35 \\
\hline Febrero & 21,41 & 92,20 & 96,81 & 75,40 \\
\hline Marzo & 21,50 & 95,50 & 100,28 & 78,77 \\
\hline Abril & 21,41 & 81,70 & 85,79 & 64,37 \\
\hline Mayo & 21,13 & 87,10 & 91,46 & 70,32 \\
\hline Junio & 20,85 & 89,20 & 93,66 & 72,81 \\
\hline Julio & 20,48 & 99,20 & 104,16 & 83,68 \\
\hline Agosto & 20,95 & 110,50 & 116,03 & 95,08 \\
\hline Septiembre & 21,23 & 111,90 & 117,50 & 96,27 \\
\hline Octubre & 21,04 & 110,00 & 115,50 & 94,46 \\
\hline Noviembre & 20,95 & 85,90 & 90,20 & 69,25 \\
\hline Diciembre & 21,04 & 86,80 & 91,14 & 70,10 \\
\hline
\end{tabular}




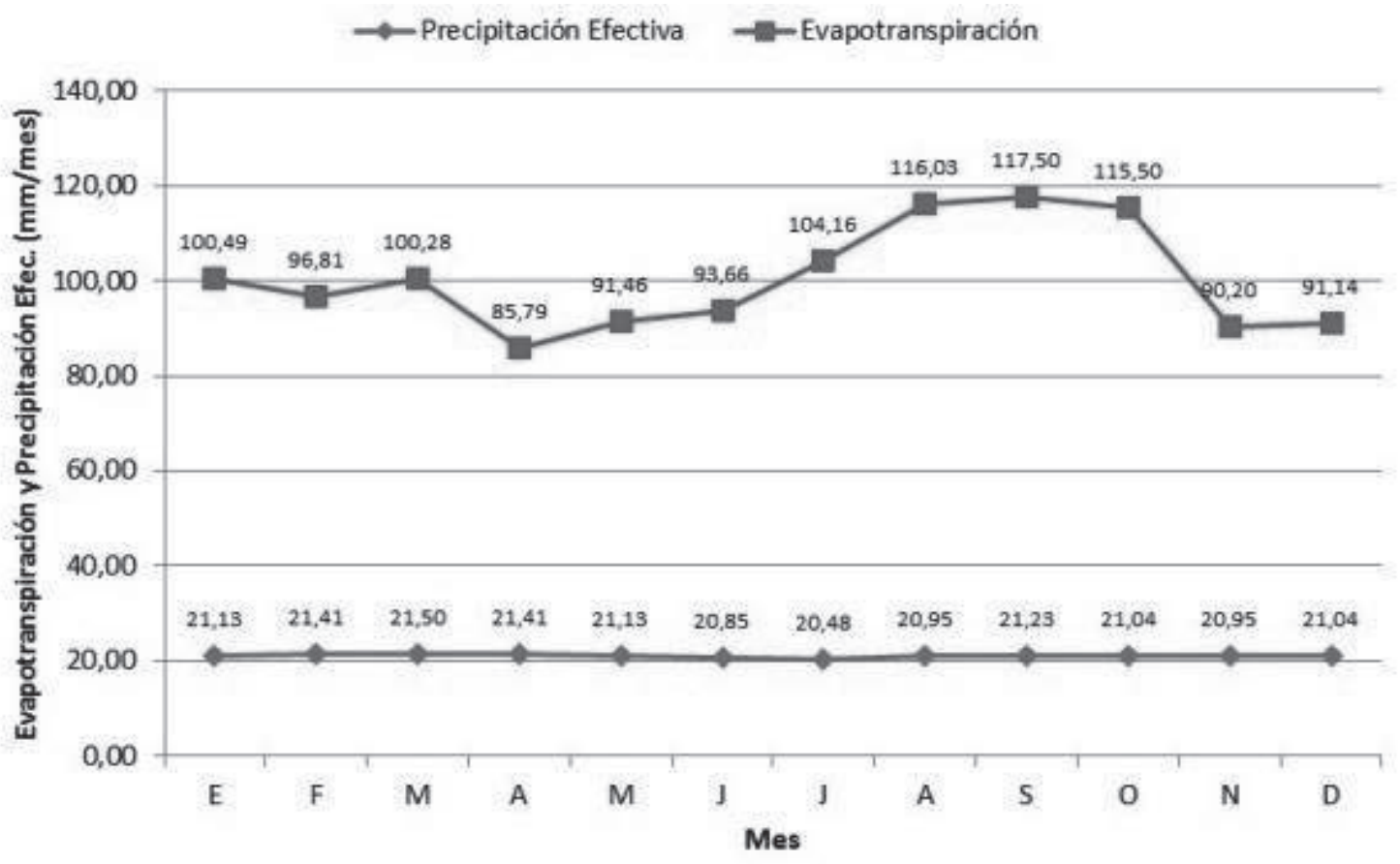

Figura 2. Balance hídrico.

los cuales, se encuentra la contaminación de acuíferos, de corrientes superficiales, de salinización del suelo; como lo indica WIKI (2007), las AR aportan sales al suelo, volviéndolo, en casos extremos, improductivo y dichos problemas se darán con más frecuencia durante los períodos cálidos, debido a la frecuencia de los riegos y al mayor consumo de agua por parte de las plantas. Se obtuvo una demanda de riego neta de $96,27 \mathrm{~mm}$ para septiembre, como dato representativo de los meses de menor pluviosidad y $68,51 \mathrm{~mm}$, como promedio de los meses de mayor pluviosidad (abril, mayo, noviembre y diciembre).

Potencialidad de Reutilización del Efluente: Como se observa en la tabla 4, el efluente de la PTAR aporta en promedio $223.720 \mathrm{~m}^{3} /$ año de agua, con los cuáles, se puede regar, en promedio, 19ha de cacao, en verano y, 27ha, en invierno; aporta además macronutrientes: $\mathrm{N}=7.273 \mathrm{~kg} / \mathrm{anno}, \mathrm{P}=$ $839 \mathrm{~kg} / \mathrm{año}, \mathrm{K}=2.797 \mathrm{~kg} / \mathrm{año}$ y lodos $958 \mathrm{~kg} / \mathrm{año}$ de materia seca, disminuyendo los costos por fertilizantes.

Los lodos, se caracterizan por presentar un alto contenido de M.O. y nutrientes que con su aplicación pueden contribuir a mejorar propiedades del suelo, pero para el adecuado uso de los lodos de plantas de tratamiento de AR, en agricultura, se hace necesario una previa caracterización física, química y biológica, que permita descartar la presencia de sustancias tóxicas y peligrosas, pues los dos principales problemas que se pueden presentar con su uso inadecuado, son los metales pesados y la presencia de microorganismos patógenos (Torres, 2002).

En conclusión, el efluente de la PTAR de Nátaga, por su calidad como agua para riego, pertenece a la clase C2S1, considerada como agua de buena calidad en irrigación y se puede usar para regar cacao. Posee peligro de salinización Medio y de Alcalinización Bajo, por lo que puede salinizar los suelos con el tiempo, si no se aplican prácticas de manejo adecuadas al cultivo y al suelo.

Por su riesgo microbiológico, el efluente pertenece a la categoría B que puede ser empleado para riego de cultivos, como cereales, industriales y forrajeros, praderas y árboles, de acuerdo con las Directrices de la OMS (2006), lo que exige un adecuado manejo del riego que minimice los riesgos de salud pública. La zona de influencia de la PTAR tiene déficit hídrico durante todo el año y el efluente se constituye en un recurso potencial para el cultivo de cacao, aportando agua $223720 \mathrm{~m}^{3} /$ año.

Con el efluente de la PTAR de Nátaga, en verano se puede regar en promedio 19 Has de cacao y en invierno 27 Has, 
Tabla 4. Potencial de Reutilización del Efluente de la PTAR de Nátaga.

\begin{tabular}{|c|c|c|c|c|c|c|c|c|c|c|}
\hline AÑO & Población & $\begin{array}{c}\text { Dotación } \\
(\mathbf{L} / \text { hab-d) }\end{array}$ & $\begin{array}{c}\mathbf{Q}_{\text {AR }} \\
(\mathbf{L} / \mathbf{s})\end{array}$ & $\begin{array}{c}\text { Vol. } \\
\text { Agua } \\
\left(\mathbf{m}^{3}\right)\end{array}$ & $\begin{array}{c}\text { Has a } \\
\text { regar } \\
\text { Verano }\end{array}$ & $\begin{array}{c}\text { Has a } \\
\text { regar } \\
\text { Invierno }\end{array}$ & $\begin{array}{c}\text { N (Kg/ } \\
\text { año) }\end{array}$ & $\begin{array}{c}\mathbf{P} \text { (Kg/ } \\
\text { año) }\end{array}$ & $\begin{array}{c}\text { K (Kg/ } \\
\text { año) }\end{array}$ & $\begin{array}{c}\text { LODOS } \\
\text { (materia } \\
\text { seca, Kg/ } \\
\text { año) }\end{array}$ \\
\hline 2010 & 2496 & 330 & 7,6 & 239674 & 20,4 & 28,8 & 7817 & 902 & 3006 & 1026 \\
\hline 2011 & 2566 & 327 & 7,8 & 245981 & 21,0 & 29,3 & 7956 & 918 & 3060 & 1053 \\
\hline 2012 & 2638 & 323 & 7,9 & 249134 & 21,2 & 29,8 & 8097 & 934 & 3114 & 1066 \\
\hline 2013 & 2712 & 320 & 8,0 & 252288 & 21,5 & 30,3 & 8241 & 951 & 3169 & 1080 \\
\hline 2014 & 2788 & 317 & 8,2 & 258595 & 22,0 & 30,9 & 8387 & 968 & 3225 & 1107 \\
\hline 2015 & 2866 & 314 & 8,3 & 261749 & 22,3 & 31,4 & 8536 & 985 & 3282 & 1120 \\
\hline 2016 & 2946 & 293 & 8,0 & 252288 & 21,5 & 30,2 & 8204 & 947 & 3155 & 1080 \\
\hline 2017 & 3028 & 274 & 7,7 & 242827 & 20,7 & 29,0 & 7884 & 910 & 3033 & 1039 \\
\hline 2018 & 3113 & 257 & 7,4 & 233366 & 19,9 & 27,9 & 7578 & 874 & 2915 & 999 \\
\hline 2019 & 3200 & 240 & 7,1 & 223906 & 19,1 & 26,8 & 7284 & 840 & 2802 & 958 \\
\hline 2020 & 3290 & 224 & 6,8 & 214445 & 18,3 & 25,8 & 7002 & 808 & 2693 & 918 \\
\hline 2021 & 3382 & 210 & 6,6 & 208138 & 17,7 & 24,8 & 6730 & 777 & 2588 & 891 \\
\hline 2022 & 3477 & 196 & 6,3 & 198677 & 16,9 & 23,8 & 6469 & 746 & 2488 & 850 \\
\hline 2023 & 3574 & 183 & 6,1 & 192370 & 16,4 & 22,9 & 6217 & 717 & 2391 & 823 \\
\hline 2024 & 3674 & 171 & 5,8 & 182909 & 15,6 & 22,0 & 5976 & 690 & 2298 & 783 \\
\hline 2025 & 3777 & 160 & 5,6 & 176602 & 15,0 & 21,2 & 5744 & 663 & 2209 & 756 \\
\hline 2026 & 3883 & 150 & 5,4 & 170294 & 14,5 & 20,3 & 5521 & 637 & 2123 & 729 \\
\hline
\end{tabular}

Abreviatura: $\mathrm{Q}_{\mathrm{AR}=}$ Caudal aguas residuales

valores que corresponden al $16 \%$ y $23 \%$, respectivamente, de las 120 hectáreas del Distrito de Riego, a pequeña escala de Nátaga "Asodriana".

Analizando los sistemas de riego disponibles para el proyecto, por condiciones topográficas (alta pendiente) y sanitarias, los sistemas de riego por superficie son descartados, por otro lado, los sistemas de riego localizados son los más adecuados, debido a que tienen el menor contacto con los regadores y el cultivo, pero se presenta inconvenientes por taponamiento de goteros y de mangueras, por el crecimiento de algas y altos contenido de sales en el AR. En este sentido y teniendo en cuenta los precedentes, se recomienda el sistema de riego por microaspersión, por ser un sistema localizado y menos exigentes en términos de filtración.
En este sentido, la propuesta de reutilización de AR es una alternativa ambiental y productiva, que introduce la concepción del suelo como agente filtrante y, a su vez, implementa un tratamiento terciario a la PTAR de Nátaga, por lo tanto, se minimiza el riesgo de contaminación de la quebrada El Pueblo y se mejora la calidad de vida de los pobladores aguas abajo de la descarga.

Conflictos de Interés: El manuscrito fue preparado y revisado con la participación de todos los autores, quienes declaramos que no existe ningún conflicto de intereses que ponga en riesgo la validez de los resultados presentados. Financiación: Este estudio fue financiado por la Universidad Surcolombiana y los autores. 


\section{BIBLIOGRAFÍA}

1. ALLEN, R.G.; PEREIRA, L.S.; RAES, D.; SMITH, M. 1998. Crop evapotranspiration-Guidelines for computing crop water requirements - FAO. Irrigation and drainage. (Italia). Paper 56. 300.

2. CIRELLI, G.; CONSOLI, S.; LICCIARDELLO, F.; AIELLO, R.; GIUFFRIDA, F.; LEONARDI, C. 2012. Treated municipal wastewater reuse in vegetable production. Agr. Water Manag. (USA). 104:163-170.

3. FASCIOLO, G.; MECA, M.; CALDERÓN, E.; REBOLLO, M. 2005. Contaminación microbiológica en ajos y suelos regados con efluentes domésticos tratados. Rev. FCA. UNCuyo. (Argentina). 37(1):31-40.

4. FOOD AND AGRICULTURE ORGANIZATION OF THE UNITED NATIONS FAO. 1999. Wastewater Treatment and Use in Agriculture. Disponible desde Internet en: www.fao.org/docrep/T0551E/T0551E00. html (con acceso el 18/10/2009).

5. FOOD AND AGRICULTURE ORGANIZATION OF THE UNITED NATIONS FAO. 2011. Reflections on reused water as a resource. Disponible desde Internet en http://www.fao.org/nr/water/news/wastewater.html (con acceso 27/01/2012).

6. HASSANLI, A.; EBRAHIMIZADEH, A.; BEECHAM, S. 2008. The effects of irrigation methods with effluent and irrigation scheduling on water use efficiency and corn yields in an Arid Region. Agr. Water Manag. 96(1):93-99.

7. JARAMILLO, B. 2008. Riegos y Drenaje. Ed, Universidad Nacional de Colombia (Palmira).420p.

8. KIZILOGLU, F.; TURAN, M.; SAHIN, U.; KUSLU, Y.; DURSUN, A. 2008. Effects of untreated and treated wastewater irrigation on some chemical properties of cauliflower (Brassica olerecea L. var. botrytis) and red cabbage (Brassica olerecea L. var. rubra) grown on calcareous soil in Turkey. Agr. Water Manag. 95:716-724.

9. LELIO, H.; REBORA, C.; GÓMEZ, L. 2009. Potencial de obtención de bioetanol a partir de topinambur (Helianthus tuberosus L.) regado con aguas residuales urbanas. Rev. FCA. UNCuyo. 41(1):123133.

10. LEÓN, G. 1995. Parámetros de Calidad para el Uso de
Aguas Residuales. Guías de Calidad de Efluentes para la Protección de la Salud. Ed. Centro Panamericano de Ingeniería Sanitaria y Ciencias Ambientales (Perú). 20p.

11. MARA, D.; CAIRNCROSS, S. 1990. Directrices para el uso sin riesgos de aguas residuales y excretas en agricultura y acuicultura. OMS (Colombia). 220p.

12. MARQUES, R.; HERPIN, U.; FERREIRA, A.; PITTOL, L.; REGINA, C.; MELFI, A. 2009. Sodicity and salinity in a Brazilian Oxisol cultivated with sugarcane irrigated with wastewater. Agr. Water Manag. 96:307-316.

13. MEDEIROS, S.S.; SOARES, A.A; FERREIRA, P.A; NEVES, J.C; MATOS, A.T; SOUZA, J.A.A. 2005. Utilização de água residuária de origem doméstica na agricultura: estudo das alterações químicas do solo. Rev. Bras. Engenharia Agr. Amb. 9(4):603-612.

14. MORATÓ, J.; SUBIRANA, A.; GRIS, A.; CARNEIRO, A.; PASTOR, R. 2006. Tecnologías sostenibles para la potabilización y el tratamiento de aguas residuales. Rev. Lasallista Invest. (Colombia). 3:19-29.

15. MUJERIEGO, R. 1990. Manual práctico de riego con agua residual municipal regenerada. Ed. Universidad Politécnica de Cataluya - UPC. 520p.

16. NUNES HENRIQUE, I.; TAVARES DE SOUSA, J.; DUARTE LEITE, V.F.; FIDELES FILHO, J.; PIRES DANTAS, J. 2005. Utilização de esgotos tratados no desenvolvimento da cultura pimentão (Capsicum annuum L.). En: Memorias $23^{\circ}$ Congresso Brasileiro de Engenharia Sanitária e Ambiental. ABES, Associação Brasileira de Engenharia Sanitária e Ambiental. Brasil. 7p.

17. O'CONNOR, T.; RODRIGO, D.; CANNAN, A. 2010. Total water management: The new paradigm for urban water resources planning. World Environm. and Water Res. Congress. Challenges of Change - Proc. the World Environm. Water Res. Congr. p.32513260.

18. ORGANIZACIÓN MUNDIAL DE LA SALUD OMS. 2006. Guidelines for the safe use of wastewater, excreta and greywater. Volume 2: Wastewater use in agriculture. Disponible desde Internet en http://www.who.int/ water_sanitation_health/wastewater/gsuweg2/en/ index.html (con acceso el 11/11/2011).

19. PESCOD, M.B. 1992. Wastewater treatment and use in 
agriculture. Irrig. Drain. (Italia). Paper 47. 400.

20. QADIR, M.; SHARMA, B.; BRUGGEMAN, A.; CHOUKRALLAH, R.; KARAJEH, F. 2007. Non-conventional water resources and opportunities for water augmentation to achieve food security in water scarce countries. Agr. Water Manag. 87:2-22.

21. RAS. 2000. Reglamento Técnico de Agua Potable y Saneamiento Básico. Disponible desde Internet en http://www1.minambiente.gov.co/viceministerios/ ambiente/dir_agua_potable_saneam_basico/ direccion/direccion_agua_potable.htm (con acceso el 18/10/2009).

22. RUTKOWSKI, T.; RASCHID, L.; BUECHLER, S. 2006. Wastewater irrigation in the developing world - two case Studies from the Kathmandu Valley in Nepal. Agr. Water Manag. 88(1-3):83-91.

23. SÁENZ, R. 1994. Introducción y uso de aguas residuales tratadas en agricultura y acuicultura: Riego y salud. Biblioteca virtual de desarrollo sostenible y salud ambiental (BVSDE). Disponible desde Internet en http://www.bvsde.paho.org/bvsair/e/repindex/repi53/ rys/rys.html (con acceso el 17/10/2009).

24. SEGAL, E.; DAG, A.; BEN-GAL, A.; ZIPORI, I.; EREL, R.; SURYANO, S.; YERMIYAHU, U. 2011. Olive orchard irrigation with reclaimed wastewater: Agronomic and environmental considerations. Agr., Ecosyst. Environm. (USA). 140:454-461.

25. SILVA, J.; TORRES, P.; MADERA, C. 2008. Reuso de aguas residuales domésticas en agricultura. Agr. Col. 26(2):347-359.

26. TORRES, E. 2002. Reutilización de aguas y lodos residuales. Disponible desde Internet en http://www. bvsde.paho.org/bvsaar/e/fulltext/gestion/lodos.pdf (con acceso el 06/04/2010).

27. UMAÑA, E. 2007. El reuso de aguas residuales para riego en un cultivo de maíz (Zea mays L.) una alternativa ambiental y productiva. Rev. La Calera. (Perú). 7(8):22-27.

28. VALENCIA, E. 2003. Uso de aguas residuales domésticas tratadas en lagunas de estabilización. Rev. Ing. Región (Colombia). 2(1):22-27.

29. VELIZ, E.; LLANES, J.; FERNÁNDEZ, L.; BATALLER, M. 2009. Reúso de aguas residuales domésticas para riego agrícola. Valoración científica. Rev. CENIC Cienc. Biol. (México). 40(1):35-44.

30. WIKI. 2007. Ingeniería de Aguas Residuales. Ed. Wiki (España). 238p.

Recibido: Marzo 8 de 2011

Aceptado: Marzo 21 de 2012 